\title{
REVIEW
}

\section{Does the Pulsatile Non-uniform Flow Matter in MR Flowmetry?}

\author{
Masataka Sugiyama $^{1,2^{*}}$, Yasuo Takehara ${ }^{1,2}$, and Shinji Naganawa ${ }^{2}$
}

\begin{abstract}
3D cine phase-contrast (4D flow) MRI is a sequence with great potential for non-invasive time-resolved 3D flowmetry at arbitrary vessel sections in various blood vessels. However, it is not widely known that the flowmetry with $4 \mathrm{D}$ flow MRI is vulnerable to pulsatile and non-uniform flow. Due to the limited spatial and temporal resolutions, averaging within the $3 \mathrm{D}$ voxel is occurring during the flowmetry. A simple solution is to avoid setting the measurement plane in the area where non-uniform flow is dominant, which is possible with an aid of streamline depictions generated by computational fluid dynamics (CFD) or 4D flow MRI data. Unlike 4D flow MRI, flowmetry in CFD simulation can use higher spatial and temporal resolution depending on computer performance; therefore, it is robust to fluctuating non-uniform flow. However, the performance of CFD simulations might be limited due to inlet conditions with low temporal resolution. Difficulty applying complex blood flow such as reflection flow from periphery may also limit accurate simulation. Caution should be taken when comparing the result of CFD simulation to that of $4 \mathrm{D}$ flow measurement.
\end{abstract}

Keywords: computational fluid dynamics, $4 D$ flow magnetic resonance imaging, non-laminar flow, turbulence, $2 D$ cine phase contrast

\section{Introduction}

3D cine phase-contrast (4D flow) MRI enables the acquisition of 3D time-resolved blood flow velocity vectors of large vessels such as the aorta to small vessels such as cerebral arteries in a non-invasive manner. 4D flow MRI is capable of time-resolved blood flow velocimetry at multiple measurement planes of arbitrary vessels. ${ }^{1,2}$

Unlike 2D cine phase contrast $(\mathrm{PC})$ that requires measurement sections fixed during the examination, retrospective flowmetry is a significant advantage of 4D flow MRI over the 2D cine PC. Since volumetric 3D flow vector data are acquired en-bloc, arbitrary measurement sections are available even after patients leave the MR suite.

Although 4D flow MRI enables the blood flow measurement at any arbitrary measurement section, operators should

'Departments of Fundamental Development for Advanced Low Invasive Diagnostic Imaging, Nagoya University, Graduate School of Medicine, Nagoya, Aichi, Japan

'Departments of Radiology, Nagoya University, Graduate School of Medicine, Nagoya, Aichi, Japan

*Corresponding author: Department of Fundamental Development for Advanced Low Invasive Diagnostic Imaging, Nagoya University, Graduate School of Medicine, 65, Tsurumai-cho, Shouwa-ku, Nagoya, Aichi 466-8550, Japan. Phone: +81-52-744-2327, E-mail: masa.sugi.4dflow@gmail.com

This work is licensed under a Creative Commons Attribution-NonCommercial-NoDerivatives International License.

(C2022 Japanese Society for Magnetic Resonance in Medicine

Received: July 15, 2021 | Accepted: December 29, 2021 be very careful not to be affected by the fluctuations of measurement in pulsatile and/or non-uniform flow. We have attempted to measure blood flow in the superior mesenteric artery (SMA) before and after a meal challenge with 2D cine PC MRI and 4D flow MRI, and to validate the results with computational fluid dynamics (CFD). However, we experienced considerable errors in blood flow measurement in each method in the SMA. In this paper, we discuss with paradox in flowmetry using 4D flow MRI through crosscomparison among 2D cine PC MRI, 4D flow MRI, and CFD.

\section{A Problem in MR Velocimetry: Inconsistent Blood Flow Patterns and Spatial Averaging}

For precise flowmetry, the segments with spatially nonuniform flow such as flow separation, non-laminar, and vortex flow should be avoided. ${ }^{3}$ Phase-contrast MRI such as $4 \mathrm{D}$ flow MRI and 2D cine PC is vulnerable to spatial averaging caused by spatially non-uniform flow (Fig. 1). Regardless of the size of vessels, previous studies have suffered from errors associated with fluctuations or inconsistency in flowmetry depending on measuring plane with non-laminar and spatially non-uniform flow. ${ }^{4,5}$ Contijoch et al. have performed a quantitative evaluation of vortex flow pattern in ascending aorta and successfully showed its correlation to the error in flowmetry. ${ }^{6}$ This type of error in flowmetry could be avoided if the voxel size is small enough. Fukuyama et al. have shown in their flow phantom 

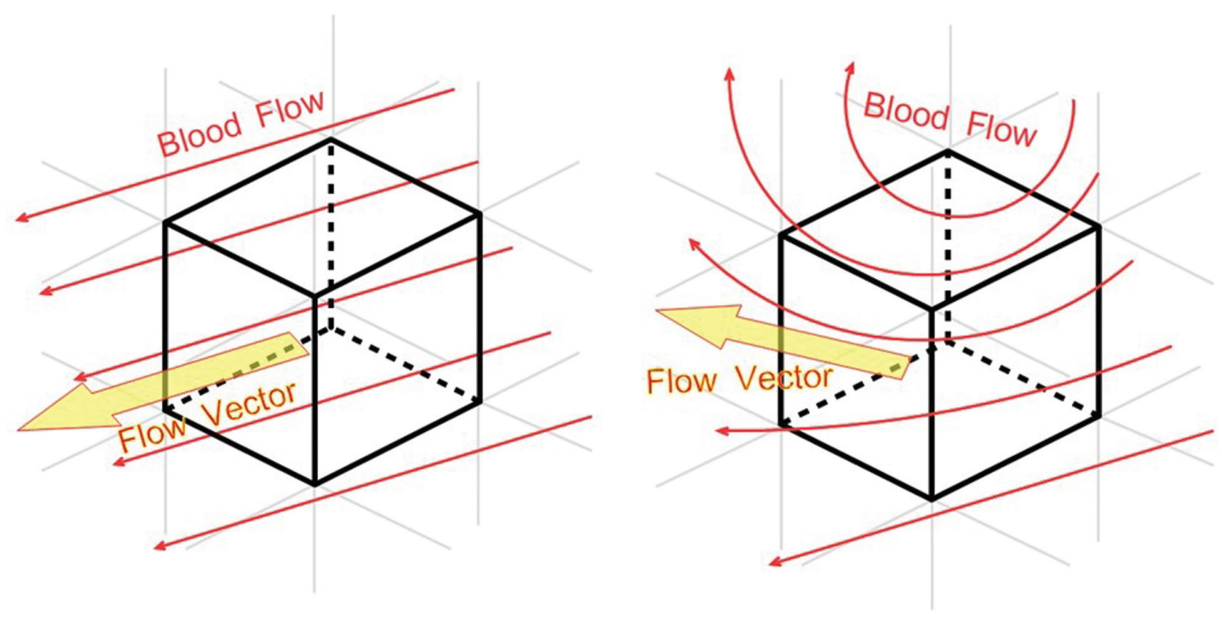

Fig. 1 Flow vector data are spatially averaged in a voxel with non-laminar flow. Substantial error in flowmetry might occur where non-laminar flow is dominant due to summation of the spatial averaging.

study that spatially averaged velocity could be measured with high accuracy when the ratio of pixel size to the diameter of vessel could be set at $30 \%$ or less. ${ }^{7}$ Spatial resolution should be carefully selected to prevent spatial averaging error in small vessels, such as cranial and abdominal visceral arteries.

Non-laminar flow patterns are likely to appear in stenotic sections, abrupt or steep curves, and/or off-branching vessels. ${ }^{8}$ Streamlines delineated from $3 \mathrm{D}$ velocity field might help select a measuring plane when velocimetry in such vessels is required; an operator can visually detect nonlaminar flow and avoid such irregular flow patterns when placing the measurement plane. The delineation of the streamline is also an advantage of 4D flow MRI over 2D cine PC since $2 \mathrm{D}$ cine PC does not allow $3 \mathrm{D}$ volumetric depictions of the blood flow.

\section{A Problem in MR Velocimetry: Temporal Averaging}

In velocimetry of pulsatile flow, temporal resolution is one of the key factors for accurate measurement in any methods. The movement of molecules in the measuring voxel with laminar flow is uniform as long as the flow is steady. However, it is different when the flow is pulsatile; the flow velocity within the voxel is no more temporally uniform. The temporally non-uniform flow vector of 4D flow MRI during one time-resolved interval is averaged. Therefore, a considerable error might occur in 4D flow MRI with low temporal resolution due to the summation of the temporal averaging. In addition, in non-laminar flow, the inconsistent flow magnitude and direction within the broad time interval are all averaged, further increasing the measurement error. The time averaging affects the accuracy regardless of whether the flow is turbulent or laminar.

A current clinically acceptable limit of time resolution of $4 \mathrm{D}$ flow MRI is generally $20-25$ phases per heartbeat, which is inferior to that of 2D cine PC with 30 phases per heartbeat. The limitation is mainly due to the acquisition time and the performance of a processor in MR Imager. On the other hand, the time resolution of CFD simulation, depending on the computer power, is even higher than that of $4 \mathrm{D}$ flow MRI or $2 \mathrm{D}$ cine $\mathrm{PC}$.

\section{A Problem in MR Velocimetry: Intravoxel Signal Dephasing}

In early study of 4D flow flowmetry, Elkins et al. have mentioned that intravoxel signal dephasing was one of the reasons for uncertainty in 4D flow flowmetry; in turbulent flow with high-order motion, the fluid molecules are mixed by eddies and induced signal dephasing and reducing SNR. ${ }^{9}$ Acceleration and spatial misalignment also cause errors in 4D flow velocimetry. Kweon et al. performed 4D flow velocimetry of non-pulsatile flow within a straight tube phantom with stenosis and observed substantial velocity underestimation in the stenotic section. ${ }^{10}$ Acceleration of molecules in the stenotic section may have caused spatial misalignment at the time of signal acquisition, which may have led to signal dephasing and underestimation.

The spatial and temporal averaging in 4D flow MRI cannot be avoided due to the fact that the encoding time of 4D flow MRI is finite. John et al. have shown that conventional 4D flow velocimetry in high-speed phantom suffered substantial displacement errors and pointed out the misregistration during encoding process as the source of error in phase encoding. ${ }^{11}$ During conventional encoding process in 4D flow sequence, spatial coordinate encoding is performed following velocity encoding. Displacement and misregistration of molecule position is enlarged as the time interval between the two encoding processes prolongs. This type of error was described as inter-encoding misregistration. If the time for one encoding process is too long, the displacement of the particles will be large, and blur will occur within the 


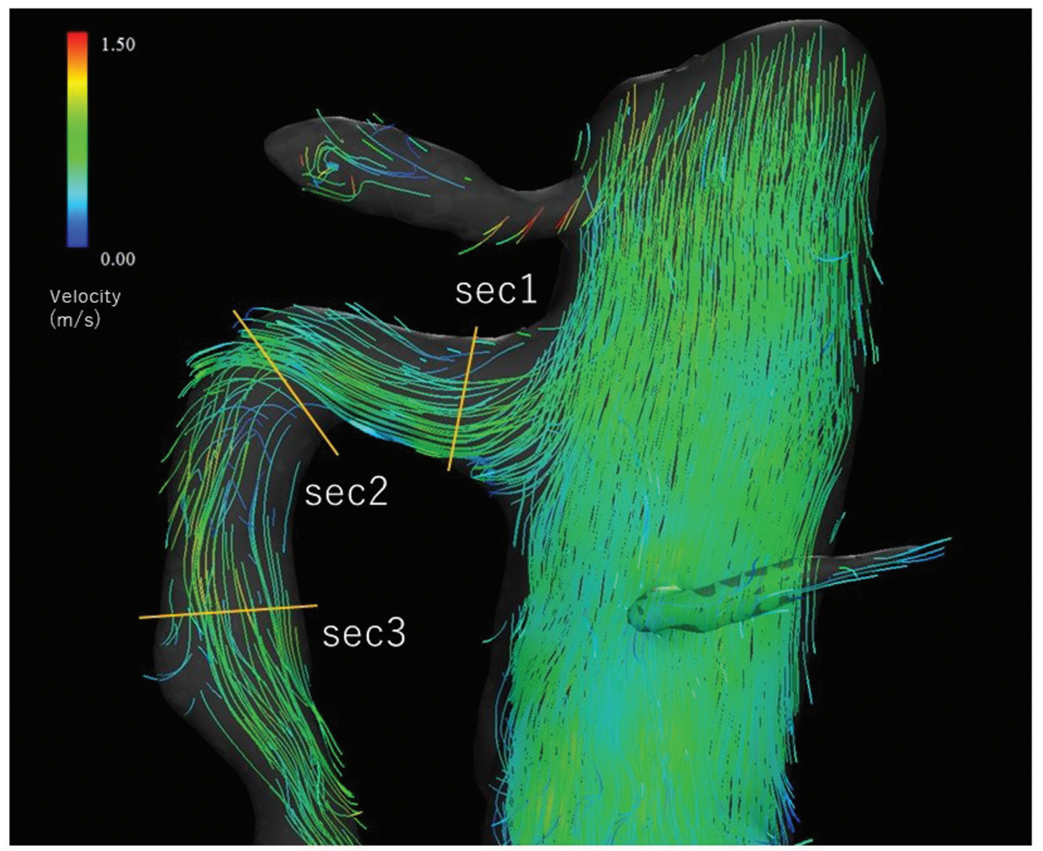

Fig. 2 The streamline of the SMA generated from $4 \mathrm{D}$ flow MRI of the 25 y.o. normal volunteer. The streamline demonstrated the nondisturbed flow in the distal section, but more vortex or helical flow due to the curvature and the acute branching in the other two sections.

encoding process. This type of error is described as intraencoding misregistration.

Short encoding time and resultant short TE are desirable for minimizing the signal dephasing due to acceleration and spatial misalignment. O'brien et al. performed non-pulsatile 2D cine PC velocimetry of non-stenotic and stenotic phantoms with various TEs and demonstrated that the shortening TE improved the relative signal ratio and reduced the intravoxel dephasing. ${ }^{4}$

\section{Case: 4D Flow MRI of the SMA of a 25- year-old Healthy Volunteer}

We would like to present one example of a 25 -year-old healthy male volunteer whose SMA was examined by 4D flow MRI, 2D cine PC, and CFD simulation. 4D flow MRI covered from the main stem branching from the aorta to the distal straight segment. Three measuring sections in the SMA (sec1: proximal, sec2: mid-curvature, sec3: distal) were set for 4D flow MRI, 2D cine PC, and CFD, and measured time-resolved flow volume at each section (Fig. 2).

Substantial uncertainty in the flow volume was observed at all three sections, especially at diastole (Fig. 3). The distal section (sec3) showed minor instability during diastole and the sharpest peak at systole. The streamline of the SMA demonstrated the most negligible vortex or helical flow in the distal section, but more nonlaminar and spatially non-uniform flows in the other two sections with a sharp curve and steep branching. The flowmetry in mid and proximal sections may have affected by signal dephasing due to spatial averaging, displacement, and misregistration errors caused by highorder motion.

The 4D flow MRI measurement was also affected by temporal averaging due to temporal non-uniformity of flow and lower temporal resolution than that of $2 \mathrm{D}$ cine PC. $4 \mathrm{D}$ flow flowmetry may also be more prone to displacement error due to its longer TE. 2D cine PC demonstrated a sharp peak at systole and deep retrograde reflection at endsystole compared to 4D flow MRI (Fig. 4). Fluctuation of flow volume was also minimal during diastole.

\section{Comparing 4D Flow MRI Velocimetry to CFD Simulation}

CFD is often used to validate 4D flow MRI research of various vessels. $^{12-15}$ However, the principles of the flowmetry between the two methods are quite different; 4D flow MRI is based on in vivo data acquisition, and CFD is based on insilico calculation. The velocimetry in vivo is a result of actual flow, reflecting all boundary conditions including wall compliance. However, CFD does not reflect these conditions.

To test if similar measurement errors occur in CFD, we have performed a simulation using the same $3 \mathrm{D}$ model of the above-shown SMA covering from the branching to the distal straight section. The geometric vessel model was generated from the magnitude image of the 4D flow MRI flow vector (Fig. 5). The inlet was set at the section in the abdominal aorta above the celiac artery, and the inlet boundary condition was set from the time-resolved blood flow rate of distal descending thoracic aorta measured from 4D flow MRI data. All outlet boundary conditions (celiac artery, SMA, bilateral 

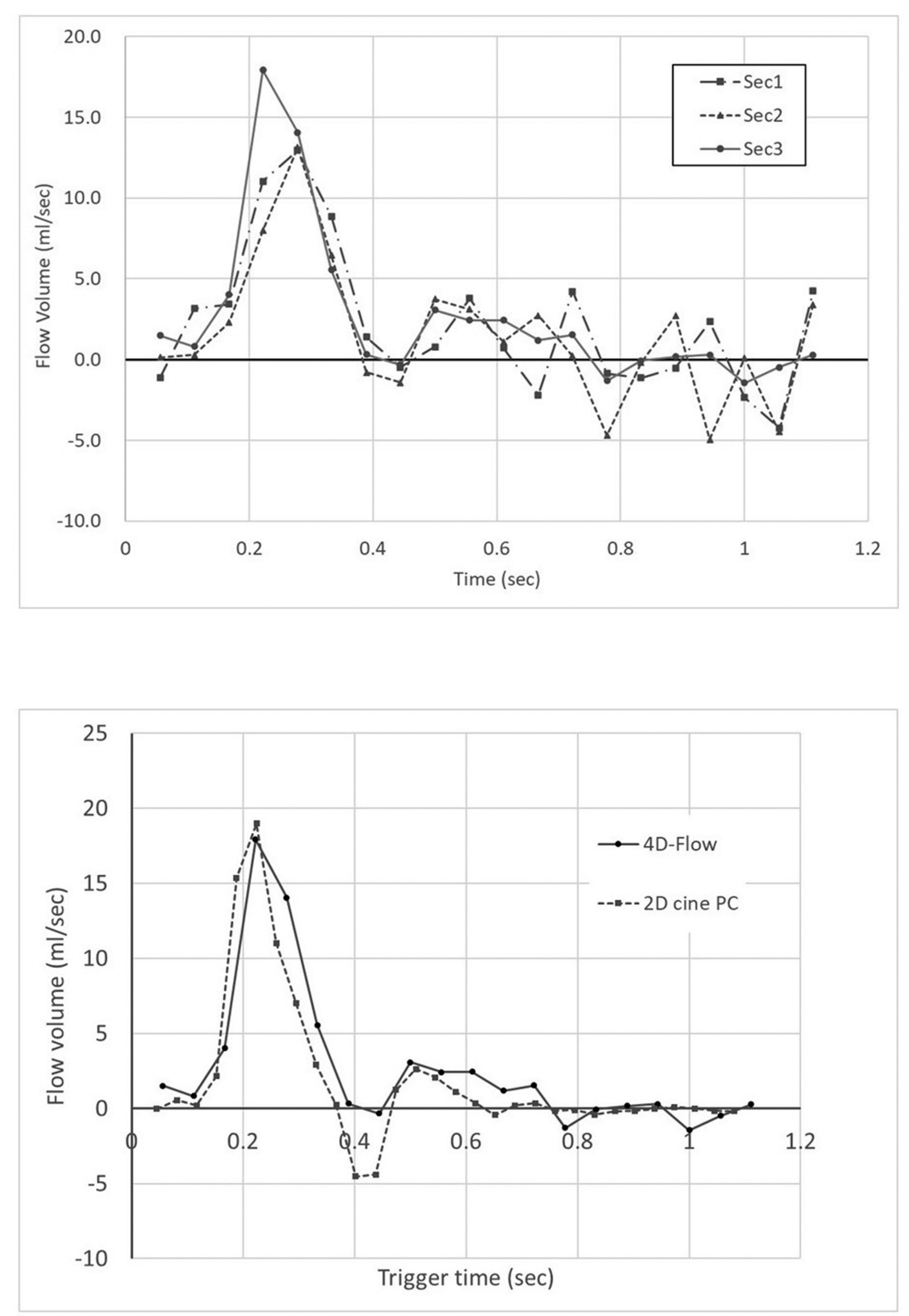

Fig. 3 The time-flow-volume curve in each section of the SMA. The distal section (sec 3 ) has the least fluctuation in blood flow during diastole and the sharpest peak at systole. SMA, superior mesenteric artery.

Fig. 4 The time-flow-volume curve at the distal section in the SMA of a 25 y.o. normal volunteer. A feasible limit of time resolution of 4D flow MRI (20-25 phases per heartbeat) is inferior to that of $2 \mathrm{D}$ cine PC (30 phases per heartbeat), which makes 4D flow MRI more vulnerable to temporal averaging. SMA, superior mesenteric artery. renal arteries, and infra-renal abdominal aorta) were set to zero pressure outlet. The standard size of polyhedra was 0.5 $\mathrm{mm}$ (approximately $1 / 10$ of the diameter of the SMA), and the time intervals were set to 0.05 (20 phases $/ \mathrm{sec})$ and 0.02 (50 phases/sec). Transient flow CFD simulation was performed for one heartbeat $(1.11 \mathrm{sec})$. The simulation was performed by solving the Navier-Stokes equations, a set of equations describing the motion of fluids in a finite volume. The simulation was based on several assumptions as follows: rigid vessel walls and an incompressible Newtonian fluid. Three measurement sections were set identically to those of 4D flow MRI measurement.

The simulation showed completely identical blood flow rates in all three measuring sections regardless of the time resolution (Fig. 6). Even the temporal resolution is set as the same as that of 4D flow MRI, the flow rate was calculated with minimal errors. The simulation was based on the finite volume method with Newtonian fluid, in which the volume of the fluid is constant. Inter-mesh delivery of blood flow was calculated by solving the Navier-Stokes equations, representing the conservation law of fluid. Since the inflow and outflow of the blood flow into and out of each mesh are calculated to be equal, the resulting macroscopic flow rate was conserved in any section of the blood vessel.

Measurement errors are minimal even in sections where non-laminar flow is dominant in CFD simulation. In fact, in the present CFD simulation of the SMA, although the streamlines demonstrated substantial helical flow at the curved middle section (Fig. 7), the flow volume of all three measurement sections showed a negligible difference. The 


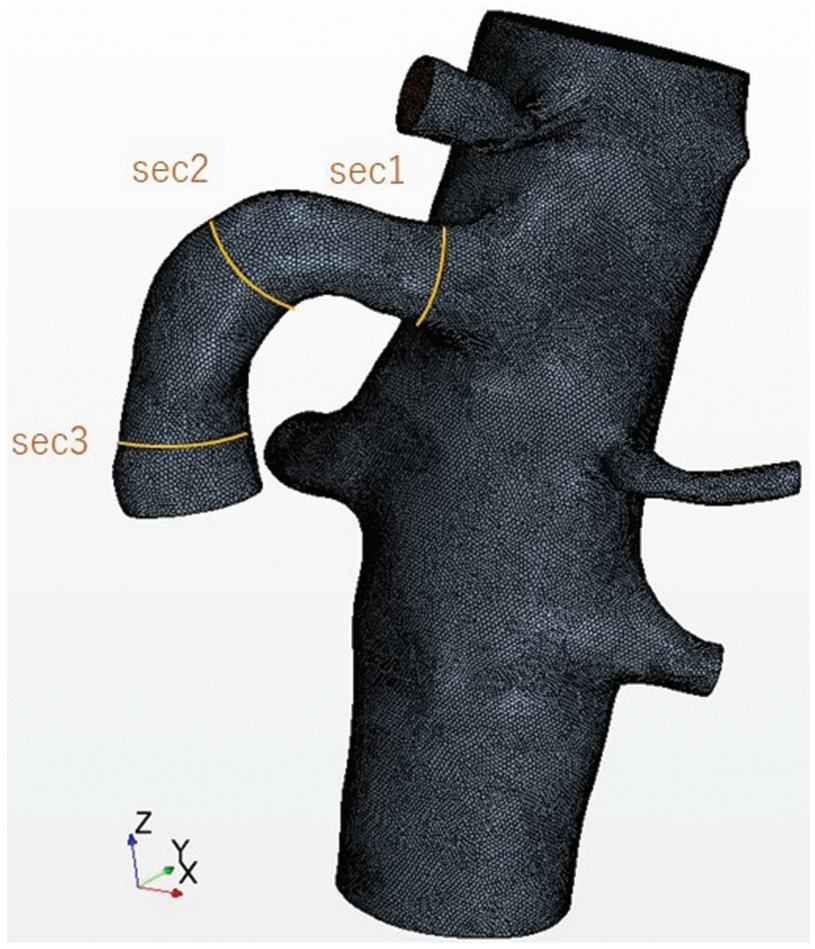

Fig. 5 CFD simulation was performed using a geometric model of the SMA generated from the 4D flow MRI. The measurement sections were set identically to those performed for the 4D flow MRI measurement. CFD, computational fluid dynamics; SMA, superior mesenteric artery.
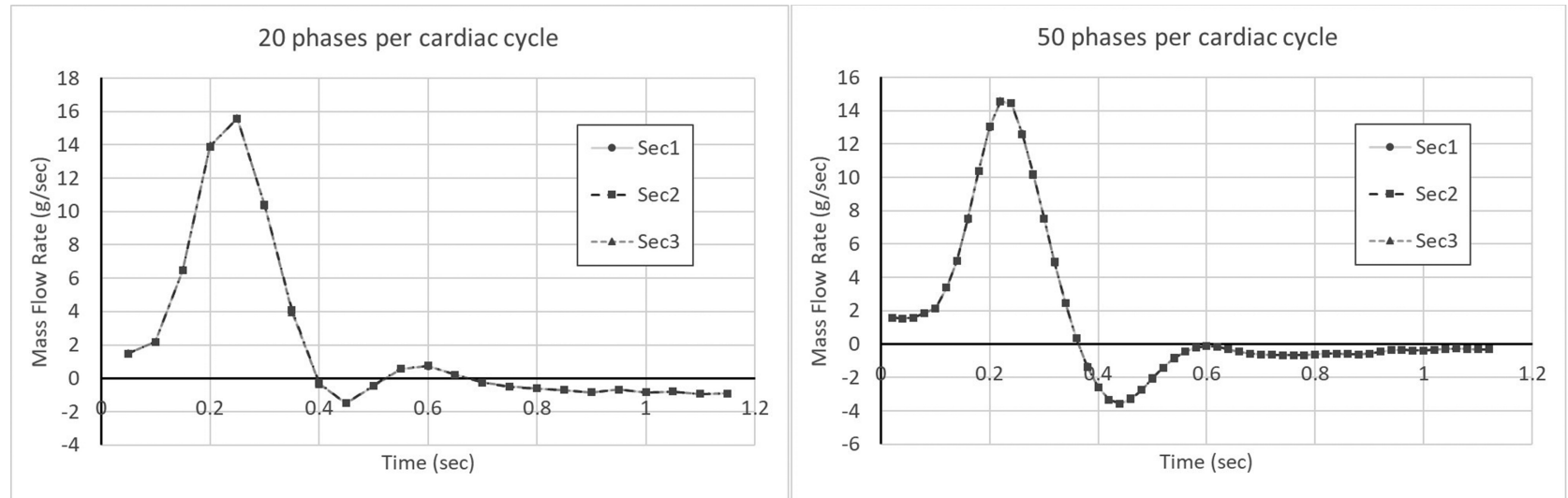

Fig. 6 The graph of the mass flow rate measured in each section of the SMA at phase resolving of 20/cardiac cycle (left) compared to the one with 50/cardiac cycle (right) calculated from the CFD simulation. The simulation showed completely identical flow rates in all three measuring sections regardless of time resolution. CFD, computational fluid dynamics.

result suggests that caution should be taken when comparing CFD and 4D flow MRI flowmetry; CFD could calculate the flow velocity and flow rate with minimal errors even in sections with non-laminar flow, whereas 4D flow MRI velocimetry is vulnerable to non-laminar flow. The flow measurement in 4D flow MRI might be inconsistent when the measuring section was set in the same manner as of CFD. Non-laminar flow should also be avoided when placing measurement planes even in CFD, if the result of $4 \mathrm{D}$ flow MRI measurement is to be compared.
In CFD analysis, it is necessary to set the inlet conditions when performing transient analysis. The inlet condition is set from flow rate obtained from the result of $2 \mathrm{D}$ cine PC or 4D flow MRI measurement. However, it should be noted that the time averaging already occurs at this point; the temporal resolution of inlet conditions is limited to that of 2D cine PC or 4D flow MRI, no matter how the temporal resolution of CFD is set fine. In addition, CFD simulation may face difficulty in applying complex blood flow. The inlet condition is supposed to be set in a plane 


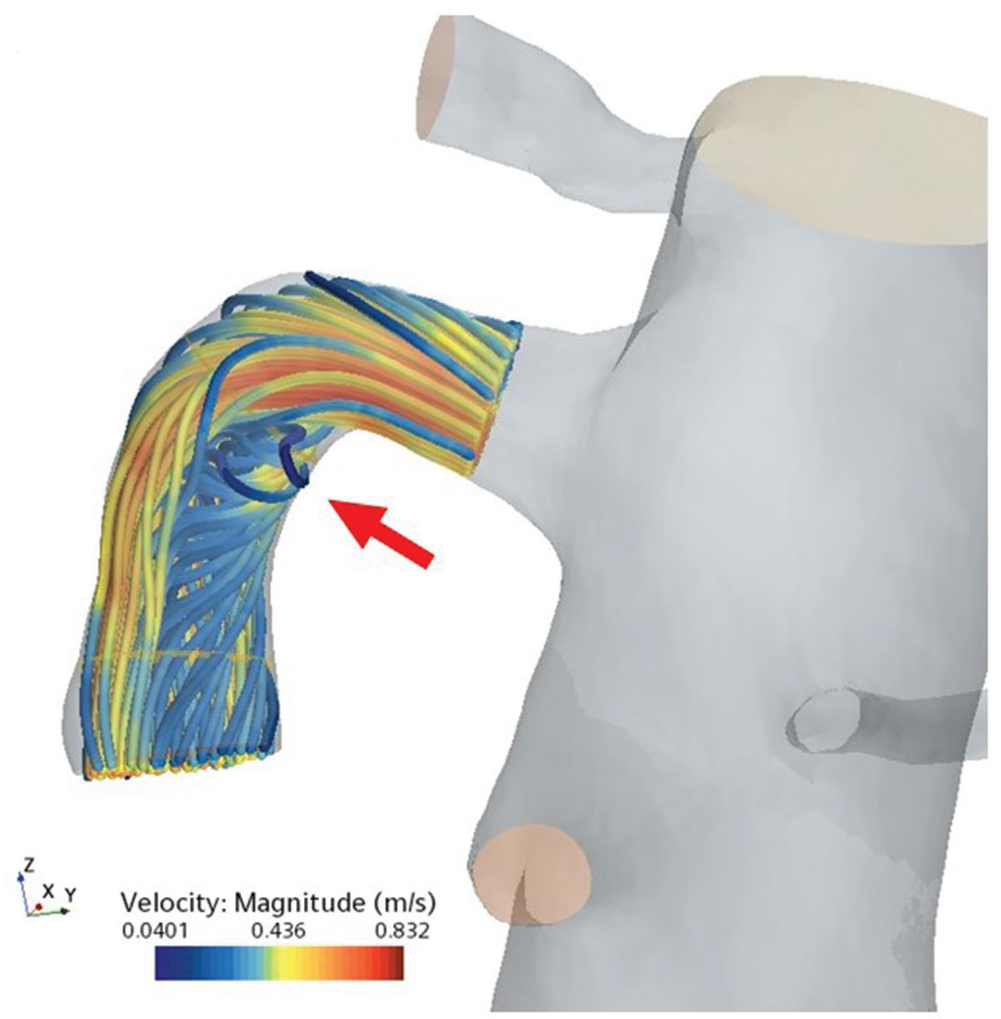

Fig. 7 The streamline of the SMA at systole generated from CFD simulation. Although substantial vortex flow appears at the mid-curved section (arrow), flow volume calculated from the simulation was almost identical to those of the other two sections. CFD, computational fluid dynamics; SMA, superior mesenteric artery. with antegrade laminar flow. The simulation hardly converges in simulations with retrograde flows, such as regurgitation and reflection flow. For these reasons, CFD does not reflect actual blood flow in vivo as 4D flow MRI does, and caution should be taken when comparing the result between each other.

\section{Conclusion}

4D flow MRI is a sequence with great potential for non-invasive time-resolved 3D flowmetry at arbitrary vessel sections in various blood vessels. However, 4D flow MRI flowmetry is vulnerable to non-laminar blood flow due to spatial and temporal averaging. Presence of non-laminar flow in measuring plane might disturb precise flow vector data acquisition due to spatial averaging. Relatively low temporal resolution also limits precise flowmetry regardless of flow pattern. Streamline, which can also be generated from 4D flow MRI vector field data, is helpful for the location of the error-prone non-laminar flow. Although CFD is robust to inconsistent non-laminar flow, the low temporal resolution of inlet condition and difficulty in applying complex blood flow may limit accurate simulation.

\section{Conflicts of Interest}

The first author Masataka Sugiyama is an endowed clinical instructor, and the second author Yasuo Takehara is an endowed chair of the same department financially supported by a private company, which is irrelevant to the contents of the paper. Shinji Naganawa has nothing to declare.

\section{References}

1. Markl M, Frydrychowicz A, Kozerke S, Hope M, Wieben O. 4D flow MRI. J Magn Reson Imaging 2012; 36:1015-1036.

2. Markl M, Chan FP, Alley MT, et al. Time-resolved threedimensional phase-contrast MRI. J Magn Reson Imaging 2003; 17:499-506.

3. Dyverfeldt P, Bissell M, Barker AJ, et al. 4D flow cardiovascular magnetic resonance consensus statement. J Cardiovasc Magn Reson 2015; 17:72.

4. O'Brien KR, Cowan BR, Jain M, Stewart RA, Kerr AJ, Young AA. MRI phase contrast velocity and flow errors in turbulent stenotic jets. J Magn Reson Imaging 2008; 28:210-218.

5. Sugiyama M, Takehara $Y$, Kawate $M$, et al. Optimal plane selection for measuring post-prandial blood flow increase within the superior mesenteric artery: analysis using 4D flow and computational fluid dynamics. Magn Reson Med Sci 2020; 19:366-374.

6. Contijoch FJ, Horowitz M, Masutani E, Kligerman S, Hsiao A. $4 \mathrm{D}$ flow vorticity visualization predicts regions of quantitative flow inconsistency for optimal blood flow measurement. Radiol Cardiothorac Imaging. 2020; 2:e190054.

7. Fukuyama $A$, Isoda $H$, Morita $K$, et al. Influence of spatial resolution in three-dimensional cine phase contrast magnetic 
resonance imaging on the accuracy of hemodynamic analysis. magn reson med sci 2017; 16:311-316.

8. Ishikawa $\mathrm{T}$, Takehara $\mathrm{Y}$, Yamashita $\mathrm{S}$, et al. Hemodynamic assessment in a child with renovascular hypertension using time-resolved three-dimensional cine phase-contrast MRI. J Magn Reson Imaging 2015; 41:165-168.

9. Elkins CJ, Markl M, Pelc N, Eaton JK. 4D Magnetic resonance velocimetry for mean velocity measurements in complex turbulent flows. Exp Fluids 2003; 34:494-503.

10. Kweon J, Yang DH, Kim GB, et al. Four-dimensional flow MRI for evaluation of post-stenotic turbulent flow in a phantom: comparison with flowmeter and computational fluid dynamics. Eur Radiol 2016; 26:3588-3597.

11. John K, Jahangir S, Gawandalkar U, et al. Magnetic resonance velocimetry in high-speed turbulent flows: sources of measurement errors and a new approach for higher accuracy. Exp Fluids 2020; 61:27.
12. Itatani K, Miyazaki S, Furusawa $\mathrm{T}$, et al. New imaging tools in cardiovascular medicine: computational fluid dynamics and 4D flow MRI. Gen Thorac Cardiovasc Surg 2017; 65:611-621.

13. Miyazaki S, Itatani K, Furusawa T, et al. Validation of numerical simulation methods in aortic arch using 4D Flow MRI. Heart Vessels 2017; 32:1032-1044.

14. van Ooij P, Schneiders JJ, Marquering HA, Majoie CB, van Bavel E, Nederveen AJ. 3D cine phase-contrast MRI at $3 \mathrm{~T}$ in intracranial aneurysms compared with patientspecific computational fluid dynamics. AJNR Am J Neuroradiol 2013; 34:1785-1791.

15. Isoda H, Ohkura $\mathrm{Y}$, Kosugi $\mathrm{T}$, et al. Comparison of hemodynamics of intracranial aneurysms between MR fluid dynamics using 3D cine phase-contrast MRI and MRbased computational fluid dynamics. Neuroradiology 2010; 52:913-920. 